\title{
ACOMETIMENTO DA SUPRA-RENAL ASSOCIADO À PARACOCCIDIOIDOMICOSE
}

\section{Leonardo Maurício Diniz', Ênio Roberto Pietra Pedroso ${ }^{1}$ e Eduardo Bambirra ${ }^{2}$}

\begin{abstract}
A supra-renal foi estudada em 60 pacientes com paracoccidioidomicose. Dentre eles, $10(16,7 \%)$ apresentavam alteraçòes anatômicas ou funcionais das supra-renais. As lesões glandulares associaram-se à paracoccidioidomicose disseminada, com evolucão da doença de pelo menos cinco anos sem tratamento e com hipotensão arterial sistêmica. Não houve associação entre alterações anatomoclínicas supra-renais e alteraçoes radiologicas pulmonares. Esses dados revelam a importância da avaliaçāo sistemática da função supra-renal em portadores de formas disseminadas da paracoccidioidomicose.
\end{abstract}

Palavras-chaves: Paracoccidioidomicose supra-renal. Blastomicose sul-americana e supra-renal. Função adrenal. Paracoccidioidomicose.

A paracoccidioidomicose (PCM) pode cursar com variado espectro de manifestações, desde micoseinfecção, a formas paucissintomáticas ou sistêmicas, com evolução protaída até rápida e ostensiva, com acometimento de múltiplos órgãos. 28

Dentre os vários órgãos acometidos, a suprarenal aparece de forma destacada. A presença de alterações supra-renais pode ocorrer em $48,2 \%{ }^{5}$ dos casos, sendo superado apenas pelo acometimento pulmonar e ganglionar. 4

A importância da integridade morfofuncional das supra-renais para a manutenção da vida é significativa. Este trabalho tem por finalidade avaliar alterações supra-renais em pacientes com PCM em função do tempo de evolução, às formas anatomoclínicas da PCM, a sintomatologia de suspeição de insuficiència supra-renal, do estado nutricional, a idade, cor, profissão dos pacientes e de seus niveis de leucócitos, linfócitos e eosinófilos sangüíneos, eletrólitos plasmáticos, glicemia de jejum, niveis de transaminases e fosfatase alcalina e telerradiografia do tórax.

\section{MATERIAL E MÉTODOS}

Foram estudados 60 individuos entre 8 e 69 anos, de ambos os sexos, com diferentes formas anatomoclinicas da PCM. Procediam dos Estados de Minas Gerais, Bahia, Espirito Santo, todos de baixo nivel sócio-econòmico. Foram procurados especificamente alteraçōes supra-renais seja pelo estudo funcional ou necroscópico.

1. Departamento de Clínica Médica.

2. Departamento de Anatomia Patológica e Medicina Legal. Faculdade de Medicina da Universidade Federal de Minas Gerais, Av. Alfredo Balena, 190 - Santa Efigenia - Cep: 30130 - Belo Horizonte - Minas Gerais - Brasil.

Recebido para publicação em 8/6/88.
Foram distribuídos em dois grupos: I) com lesão supra-renal (clinica, funcional ou anatomopatológica) e II) sem evidência de lesão supra-renal (clínica, funcional ou anatomopatológica).

\section{RESULTADO}

O grupo com lesão supra-renal foi constituído por 10 pacientes (Tabela 1). Sete foram diagnosticados após estudo anatomopatológico em pacientes necropsiados. Dai em diante seus prontuários foram analisados retrospectivamente. Em 3 pacientes foi diagnosticada insuficiència supra-renal em vida, após estudo funcional alguns anos após a cura clínica da PCM. Também seus prontuários foram analisados retrospectivamente.

O grupo sem lesão supra-renal foi constituido por 50 pacientes (Tabela 1) internados consecutivamente para esclarecimento diagnóstico e encontrado PCM. Foram analisados retrospectivamente.

Os métodos utilizados na avaliação das condições clínico-laboratoriais, radiológicas e anatomopatológicas de ambos os grupos foram constituidos por: a) avaliação clínica: busca de alteraçōes compativeis com insuficiência supra-renal como escurecimento da pele, pigmentaçāo anormal de mucosas visíveis, hipotensão, labilidade hidroeletrolitica, taquicardia reflexa em ortostatismo, manifestaçōes sugestivas de hipoglicemia e astenia intensa; b) avaliação laboratorial: constou de ionograma, glicemia, hemograma, leucograma, transaminases e fosfatase alcalina séricas. A avaliação direta da função supra-renal nos pacientes vivos constou da dosagem do cortisol durante crise addisoniana ou dosagem dos niveis basais $\mathrm{e}$ pós-estimulo (corticotrofina sintética) do cortisol plasmático, sem crise de insuficiència manifesta. Foi tambem realizado o teste de sobrecarga hidrica. Nos pacientes estudados após necropsia foram realizados 
Diniz LM, Pedroso ERP, Bambirra E. Acometimento da supra-renal associado à paracoccidioidomicose. Revista da Sociedade Brasileira de Medicina Tropical 21: 173-176, Out-Dez, 1988

Tabela I - Distribuicão dos pacientes dos grupos com e sem lesōes supra-renais de acordo com a faixa etária, sexo e cor.

\begin{tabular}{|c|c|c|c|c|c|c|c|c|c|c|}
\hline \multirow[t]{2}{*}{ Grupos } & \multicolumn{3}{|c|}{$\begin{array}{c}\text { Idade } \\
\text { (anos) }\end{array}$} & \multicolumn{3}{|c|}{ Sexo } & \multicolumn{4}{|c|}{ Cor } \\
\hline & $<20$ & $\geq 20$ & $\mathrm{~T}$ & $\mathbf{M}$ & F & $\mathrm{T}$ & $\mathrm{L}$ & $\mathrm{Fa}$ & $\mathrm{Me}$ & $\mathrm{T}$ \\
\hline I & 4 & 6 & 10 & 6 & 4 & 10 & 2 & 5 & 3 & 10 \\
\hline II & 20 & 30 & 50 & 38 & 12 & 50 & 19 & 23 & 8 & 50 \\
\hline Total & 24 & 36 & 60 & 44 & 16 & 60 & 21 & 28 & 11 & 60 \\
\hline
\end{tabular}

M: masculino: F: feminino: L: leucodermo: Fa: faiodermo: Me: melanodermo; $T$ : total

cortes histológicos das supra-renais, corados pela hematoxilina-eosina e prata-metenamina de Gomori; c) avaliação radiológica: telerradiografia do tórax em PA e perfil.

Excluiu-se a concomitància de outras entidades mórbidas capazes de produzir lesōes nas supra-renais e passiveis de diagnóstico diferencial como tuberculose, linfomas, câncer metastático, meningococcemia, uso de medicamentos, septicemias.

O nivel de significància mínimo aceitável foi de $5 \%$.

\section{RESULTADOS}

Dentre os 60 pacientes, 10 apresentavam alteraçōes supra-renais devidas a PCM $(16,7 \%)$.

Não houve correlação significativa entre alteraçōes da funçào supra-renal relacionadas à PCM e faixa etária, sexo, cor, profissão. Em $90 \%$ dos casos, a PCM com ou sem lesão supra-renal ocorreu em pacientes após os 12 anos de cidade, sendo que $30 \%$ até os 20 e $60 \%$ após os 20 anos de idade. A distribuição entre os sexos masculino e feminino foi de $3: 2$, respectivamente, nos dois grupos independentemente de terem ou não lesão supra-renal.

$O$ estado nutricional não se correlacionou com a presença ou não de alterações supra-renais, entretanto, a desnutriçảo esteve presente em $80 \%$ dos pacien- tes com lesão supra-renal e em $50 \%$ nos sem lesão supra-renal.

Houve associação significativa $(p<0,01)$ entre a medida da tensão arterial (hipotensão) e alterações anatomoclínicas supra-renais, independentemente de outro parâmetro clinico.

A relação entre alterações anatomoclinicas da supra-renal e a evolução clínica da PCM, apresentou as seguintes correlações significativas: PCM disseminada desde o início de sua apresentação clínica e presença de alterações anatomoclinicas paracoccidioidomicóticas na supra-renal $(\mathrm{p}<0,01)$ alterações anatomopatológicas supra-renais paracoccidioidomicóticas e forma disseminada da PCM no momento em que o diagnóstico foi realizado $(p<0,05)$, presença de alterações anatomopatológicas supra-renais e o período de tempo entre o inicio da doença e o diagnóstico da PCM, sendo este intervalo igual ou superior a cinco anos ( $p>0,01)$ (Tabela 2).

Não houve correlação significativa entre presença de alteraçōes anatomoclínicas supra-renais e eosinófilos sangüineos, eletrólitos plasmáticos (sódio/potássio), glicemia de jejum ou transaminases.

Houve correlação significativa entre linfocitopenia $(\mathrm{p}<0,01)$ ou fosfatase alcalina elevada $(\mathrm{p}<$

Tabela 2 - Alterações anatomo-clinicas da supra-renal em pacientes com PCM em função da evoluçào da doença.

\begin{tabular}{|c|c|c|c|c|c|c|}
\hline \multirow{3}{*}{$\begin{array}{l}\text { Áiteraçòes da } \\
\text { supra-renal }\end{array}$} & \multicolumn{6}{|c|}{ Evolução } \\
\hline & \multicolumn{2}{|c|}{ forma inicial } & \multicolumn{2}{|c|}{ forma atual } & \multicolumn{2}{|c|}{ tempo (anos) } \\
\hline & 1 & $\mathrm{~g}$ & 1 & $\mathrm{~g}$ & $<5$ & $\geq 5$ \\
\hline Presente & NS & $\mathrm{S}$ & NS & $S^{*}$ & NS & $\mathrm{S}$ \\
\hline Ausente & NS & NS & NS & NS & NS & NS \\
\hline
\end{tabular}


Diniz LM, Pedroso ERP, Bambirra E. Acometimento da supra-renal associado a paracoccidioidomicose. Revista da Sociedade Brasileira de Medicina Tropical 21: 173-176, Out-Dez, 1988

$0,05)$ e presença de alterações anatomoclínicas das supra-renais.

Não houve associação entre a presença de alterações radiológicas pulmonares e alterações anatomoclínicas das supra-renais devidas à PCM.

Nos três pacientes diagnosticados em vida, dois o foram alguns anos após a cura clínica da PCM. No terceiro, com o paciente em uso de sulfadiazina, teve seu curso evolutivo modificado três meses após uso de anfotericina B, com aumento do cortisol após injeção de ACTH, compativel com recuperação funcional da glândula.

Em sete pacientes do grupo I a histopatologia mostrou focos de infecção paracoccidioidomicótica das supra-renais, sendo múltiplos e numerosos em dois casos. Foram observados, concomitantemente, focos de necrose com hipotrofia do parênquima glandular.

\section{DISCUSSÃO}

A insuficiência supra-renal na PCM apareceu em $16,7 \%$ dos casos. A casuística referente à alteração supra-renal na PCM é variada indo desde $5 \%^{3}$ a $25 \%^{1}, 47 \%^{5}$ a $48,3 \% 5$. A diferença entre as casuísticas pode-se dever a dimensão das amostras e ao fato da investigação ter sido feita em material de necrópsia previamente selecionado. Esses dados revelam a importância do problema em nosso meio e, conseqüentemente, a importància da avaliação sistemática da função supra-renal em portadores de formas disseminadas da PCM, sobretudo se de longa evolução.

Os achados histopatológicos estão, em parte, de acordo com a literatura ${ }^{5}$, no entanto, há menor incidência de lesões necrosantes (gomóides) com predomínio de forma difusa, considerada rara.

Entre os très casos diagnosticados em vida, em dois observou-se insuficiència supra-renal como manifestação tardia da doença. No outro, o uso de anfotericina B resultou aumento do cortisol pós-ACTH compatíveis com recuperação funcional da glàndula.

Não foi observada diferença significativa entre pacientes com ou sem insuficência supra-renal no que se refere à faixa etária, sexo, cor. No entanto, è expressivo o número de pacientes com PCM diagnosticada após 12 anos de idade, sugerindo periodo de incubação prolongado. 569

A presença da hipotensão arterial, o encontro da forma disseminada da doença desde o início de sua apresentação clínica ou no momento em que foi feito seu diagnóstico fazem suspeitar do acometimento da supra-renal pelo $P$. brasiliensis. A longa evolução de doença ativa e progressiva, em individuos com resistència natural comprometida, aumenta as possibilidades de disseminação da doença. Considerando-se que o acometimento das supra-renais é resultado, em especial, de disseminação hematogènica ${ }^{6}$ è razoável admitir que, quanto mais prolongado o curso da doença, mas freqüente sua disseminação, maior acometimento glandular. 210

Não houve associação entre alterações suprarenais, e níveis dos eosinófilos sangüineos, sódio e potássio séricos e glicemia de jejum. A interpretação dos achados hematológicos é complexa ${ }^{5}$ e parece não seguir padrōes conhecidos da insuficiência suprarenal. A impressão é que a PCM deve exercer, por si, maior repercussão sobre o sistema hematopoiéticoimunológico que a insuficiência supra-renal isolada. ${ }^{7}$

Altos niveis de fosfatase alcalina foram encontrados entre portadores de lesões supra-renais. Esse achado pode se associar à presença de lesões hepáticas ou obstruções extra-hepáticas no curso das formas disseminadas. No entanto, niveis normais de transaminases, repetidamente encontrados, tornam essa relação menos provável. Outra possibilidade è a coexistência de lesões ósseas que freqüentemente são múltiplas, ativas e extensas, que poderiam ser responsáveis pelo aumento da fosfatase alcalina.

De forma diferente ao observado por Del Negro ${ }^{5}$, não se observou relação entre presença de lesões supr-renais e alterações radiológicas presentes à telerradiografia do tórax. A pequena dimensão da amostra pode explicar essa aparente divergência, entretanto, pode-se dissociar o acometimento supra-renal do pulmonar, relacionando ao $P$. brasiliensis tropismo especial para a supra-renal após disseminação hematogênica.

\section{SUMMARY}

Sixty patients with paracoccidioidomycosis (PCM) were studied, with special regard to adrenal involvement. Ten patients $(16.7 \%)$ had adrenal abnormalities associated with hypotension, a disseminated form of the disease and more than five years of evolution without treatment. No statistical association between adrenal anatomo-clinical alterations and radiological pulmonary abnormalities was $o b-$ served. These data point out to the importance of systematic evaluation of adrenal function in patients with disseminated PCM.

Key-words: Paracoccidioidomycosis. South American Blastomycosis. Adrenal function. Adrenal failure.

\section{REFERÈNCIAS BIBLIOGRÁFICAS}

1. Assis LM, Bloise W, Coelho Netto AS, Burattini EL, Gueiros MFM, Epps DR, Borelli A, Flosi AZ, Cintra ABU. Oito casos de doença de Addison. A blastomicose sul-americana como possível fator etiológico em dois. Revista da Associação Médica Brasileira 5: 56-71, 1959. 
Diniz LM, Pedroso ERP, Bambirra E. Acometimento da supra-renal associado à paracoccidioidomicose. Revista da Sociedade Brasileira de Medicina Tropical 21: 173-176, Out-Dez, 1988

2. Borelli D. Some ecological aspects of Paracoccidioidomycosis. In: Pan American Symposium on Paracoccidioidomycosis, 1 st Proceedings, Washington, PAHO, p. 59-64, 1972.

3. Castro RM, Del Negro G. Particularidades clinicas da paracoccidioidomicose na criança. Revista do Hospital das Clinicas da Faculdade de Medicina de Sāo Paulo 31: 194-198, 1976.

4. Costa UP, Mendes TIA, Scherman J. Sindrome de Addison associada à blastomicose sul-americana (apresentaçāo de três casos). Revista Brasileira de Medicina 29: 224-228, 1972.

5. Del Negro G. Localização supra-renal da blastomicose sul-americana. Tese de Livre-Docència. Faculdade de Medicina da Universidade de São Paulo, São Paulo, 1961.

6. Franco MF, Montenegro MRG. Anatomia Patológica. In: Del Negro G, Lacaz CS, Fiorillo A (ed). Paracoccidioi- domicose, Sāo Paulo, Sarvier-Edusp, p. 97-117, 1982.

7. Frankel JK. Pathogenesis of infection of the adrenal gland, leading to Addison's disease in man: the role of corticoids in adrenal and generalized infection. Annals of New York Academy of Science 84: 391-440, 1960.

8. Lacaz CS, Zamith VA, Del Negro G, Siqueira AM. Aspectos clinicos gerais. Formas polares da paracoccidioidomicose. Particularidades clinicas infanto-juvenis. In: Del Negro G, Lacaz CS, Fiorillo AM. (ed) Paracoccidiojdomicose. São Paulo, Sarvier-Edusp,p. 141-147, 1982.

9. Londero AT, Ramos CD. Paracoccidioidomycosis. A clinical and mycological study of 41 cases observed in Santa Maria, RS, Brazil. American Journal of Medicine 52: 771-775, 1972.

10. Medoff G, Kabayasgi GS. Strategies in the treatment of systemic fungal infections. New England Journal of Medicine 302: 145-155, 1980. 